\title{
Brief Analysis of Social Capital Application in Community Participatory Governance from the Perspective of NIMBY
}

\author{
Honglu Fan ${ }^{1}$, Lin $\mathrm{Lu}^{1}$, Yin Zhang ${ }^{1,2}$ \\ ${ }^{1}$ School of Culture and Social Development Studies, Southwest University, Beibei, Chongqing, China \\ ${ }^{2}$ Renewable Resources Department, University of Alberta, Edmonton, AB, T6G 2C7, Canada \\ Correspondence: Lin Lu, School of Culture and Social Development Studies, Southwest University, Beibei, Chongqing, \\ China.
}

Received: November 3, 2015

doi:10.11114/smc.v3i2.1208
Accepted: November 9, 2015 Online Published: November 20, 2015

URL: http://dx.doi.org/10.11114/smc.v3i2.1208

\begin{abstract}
Social governance is a hot topic in today's society. From the "social management" to "social governance", the latter more embodies the diversity of participation, which means based on an equal partnership, government, social organizations, enterprises, communities, individuals and other actors regulate and manage social affairs, social organizations and social life according to the law, and ultimately maximize the public interest. Community is a place where social situation, public opinion, and social basic contradictions and problems are centrally reflected, so it is an important part of society, the most basic content of the social organism and the epitome of macro society. Thus, the important part of social governance is the community governance. At the same time, NIMBY (Not In My Back Yard) is the public's reaction to the event and the problem in the social governance. Lots of things from that have reference significance for the community governance. Therefore, this paper, from the perspective of NIMBY, explores how to use social capital and maximize the benefits by referring Chinese and other countries' successful experience via literature research, case analysis and comparative method, so as to better achieve the community participatory governance in China.
\end{abstract}

Keywords: NIMBY, social capital, trust, community governance, participatory governance

\section{Introduction}

\subsection{Background}

The third plenary session of the 18th central committee of the CPC pointed out making innovations in social governance. General Secretary Xi Jinping stressed that in order to strengthen and make innovation in social governance, the key is institutional innovation and the core is people. Only if people live in harmony with each other, society will be stable and orderly. The focus of social governance must be the urban and rural communities. If community service and management ability are strengthened, the foundation of social governance will be stable. Therefore, community governance is an important part of social governance. Now the society is a "risk type" "development type" and "governance type" society. Social problems continue to appear, which have many "burning point" and low "burning point". NIMBY reflects the importance of public participation and social capital. Hence, through the perspective of NIMBY, explore the significance of social capital in the process of community participatory governance and effective ways of putting social capital into community participatory governance, so as to better achieve community governance and social governance.

\subsection{Methods}

In this paper, we used literature research, case analysis and comparative method to study the issue. Specifically, the research ideas were as follows: first, through the research of Chinese and other countries' literature, we summarized present research achievements, found the research field which had not been studied, and explored the possibility of cross research. Then, we analyzed and studied Chinese and other countries' cases, summarized their advantages and disadvantages, concluded their common experience by comparison and analysis in order to learn from them. In the end, we drew a conclusion and put forward relevant suggestions for the research.

Among them, literature research method was the basis of the research, which helped the selection of the theme, 
perspective, etc. of this paper. Through the literature research method, the appropriate theoretical support also could be found for the entire article. The case analysis method through the selection of the three representative countries reflected various experience of solving the problem of NIMBY under different social backgrounds. It analyzed the problem from internal perspective, which belonged to the micro level. However, through the contrast analysis of the three cases, comparative method made up for the narrow view of the case study and the one-sided view. It was conducive to the comprehensive extraction of the common features of the cases, and provided theoretical support for the research from the macro level. In a word, these two research methods complemented each other and played significant but distinctive roles in the study.

\subsection{Literature Review}

Research on community governance was mainly in the following areas.

1) Researches on community governance model. Liu Xianjing (2006),Wang Fang \& Li Hezhong (2008), Dong Xiu (2010),Zhu Renxian \& Wu Wenying (2014), Wang Zhenzhen (2014) discussed community governance model from the different dimensions, such as the community governance of multivariate subjects participation, domestic and international comparative study, urban and rural community governance model, the change of community governance model and so on. It could be seen that scholars used the horizontal, vertical and other diversified perspective to explore the community governance model, which brought new idea for our research.

2) Researches on community governance structure. Wei Shu (2008) thought community governance structure was divided into three kinds of types - traditional community, cooperative community and administrative community. Sui Yujie (2014) thought there were function counterpoint type, demand satisfaction type and self-dependence type. Wu Zaizai \& Qian Wenhua (2014) thought community governance structure model was "government of two levels, management of three levels, and network of four levels". Besides, Liu Weihong (2008), Wang Wei (2009), and Kang Xiaoqiang (2012) researched community governance structure transformation from different sides. However, most of them discussed community governance structure from a macro perspective, while the specific community governance measures were mentioned less.

Regarding the cross research of these themes involved in this paper, there were several aspects as follows.

3) Researches on community governance and citizen participation. Jiang Xiaoping \& Heng Xia (2007), Xia Xiaoli (2014) thought citizen participation was the essential requirement of community governance, and it was the core content of political construction of democracy at community in urban area of China. It was the realization of the modern democracy in microcosmic level. Wu Jiashun (2011), Zheng Congming \& Hu Shuying (2011) discussed the necessity, characteristics, principles and specific contents of citizen participation in community governance. Scholars recognized the importance of citizen participation in community governance, but most still used many traditional ways in aspects of the specific measures for community participatory governance, which was a lack of consideration of new things and combination with hot topics in current social development. Thus it led to the lack of innovation and the implementation.

4) Researches on NIMBY and community governance. Li Zhaozuo (2013) and Wang Han (2014) thought the management of NIMBY could not simply rely on the method of economic compensation. The social factors that were reflected by NIMBY were valuable to the future social governance. Zhu Tian (2014) put forward the problem of NIMBY and public participation in the times of social governance, emphasizing that public participation was an important factor to solve the conflicts and realize social governance. Chu Cheng, Pan Jinzhu, \& Xia Meiwu (2014) explored the aspects of idea, system and mechanism, and presented the way to deal with NIMBY. Scholars saw the link between NIMBY and community governance, which provided a new perspective for our research but was short of further in-depth study of the two. The majority of researches focused on the treatment of NIMBY, but the experience of community governance from NIMBY was not further summarized, which was the problem we should study.

5) Researches on social capital and community governance. Qian Haimei (2011), from the point of view of resource allocation, explored the operation mechanism of social capital in the community governance and stressed the importance of trust in social capital and community construction. From the perspective of the failure of community governance, Shi bin \& Wu Xinxin (2009) put forward a proposal, which was cultivating social capital and promoting community governance. In the aspect of innovative community governance path, Yan Jirong (2010), He Zuocheng (2011), Guo Lida \& Yang Guihua (2014) proposed ways to provide new ideas for community governance, which were multi-subject participation in governance, construction of "acquaintance society", and promoting the development of community autonomy organization, community network construction, virtual community governance, etc. .

Here were some other countries' literature reviews.

6) Researches on NIMBY. In the history, NIMBY movement appeared in western countries with industrial civilization development, and first appeared in the United States. O 'Hare, an American scholar, first proposed the concept of 
NIMBY (1977). Emilie Travel Livezey, a British journalist, first gave the name of the term-NIMBY (not in my back yard) in the "Christian Science Monitor" on Nov.6, 1980 to describe people's aversion and revolt to chemical waste, which has been widely used by the academic circle afterwards. On the macro level, the research of the western academic circles has experienced two different stages. The division standard of different stages was the different nature of NIMBY. The first stage was from the 1970s to the beginning of the 1990s. This period was mainly based on the opposition to pollution facilities, such as dumps (Halstead, Luloff, \& Myers, 1993), especially toxic waste disposal sites (Bryant \& Mohai,1992). The second stage was from the 1990s until now, this stage was mainly based on the opposition to "non-polluting facility", such as land-use patterns (Kotsopoulos, 2000), construction of drug treatment centers and tramp shelters (Shanoff, 2000), psychiatric services (M.C Angermeyer, H Matschinger, A.Holzinger, M.G.Carta, \& G.Schomerus,2013), large-scale solar energy development (Carlisle Juliet E., Kane Stephanie L.,Solan David, Bowman Madelaine,\& Joe Jeffrey C.,2015),etc..

7) Researches on reasons analysis of NIMBY. Some scholars standing on the "economic man" hypothesis, believed financial compensation was the core factor which affected NIMBY (Kunreuther, Fitzgerald, \& Aarts,1993). Some scholars thought that increased environmental awareness and rights awareness played an important role (M. E. Vittes, P. H. Pollock, \& S. A. Lilie, 1993; Ruth, 2000, etc.).Some scholars believed that people's conflict degree for the NIMBY facility was subject to the effect of public risk perception (Robin Gregory, 1990). Some scholars believed that NIMBY was related to the development level of community economy (Lindén Andreas, Rapeli Lauri, \& Brutemark Andreas, 2015), while some scholars held that community played an important role in NIMBY (Simona Bigerna \& Paolo Polinori, 2015).

8) Researches on social capital. Social capital was first proposed by Bourdieu Pierre in 1980. It consisted of two relative but different perspectives. One of them was that individual was the center of social capital. This viewpoint was held by sociologist Burt, Nan Lin and Portes. They mainly concerned resources such as information, ideas, supports, etc., which could be obtained through contact with others. Another one was that society was the center of social capital. Coleman, Putnam and Fukuyama as representatives paid attention to the nature and extent of public participation in various informal social networks or formal civic associations. "Same as other forms of capital, social capital is productive. That people own social capital determines people can achieve certain goals, and vice versa." Coleman said in a book named "The Foundation of Social Theory". The politics meaning of social capital was put forward by Putnam. He highly summarized the concept of social capital in a book named "Making Democracy Work: Civic Traditions in Modern Italy". He thought it was civil participation network, as well as reciprocity and trust norms embodied in the convention. He also said "social capital is an important decision factor of democracy progress." Later scholars studied the important role of social capital in community governance (Paul J. Hensel, Pamela A.Kennett-Hensel, \& Julie Z. Sneath 2013; Tammy Findlay, 2014). Previous scholars enriched the research results, which provided a powerful theoretical support for the later research, and also let the theory play an important practical value.

9) Researches on community governance. Corinne Damlamian(2006); Maria E. Bobenrieth \& Darian Stibbe (2010),Jeff Hoffman (2011) explored community governance starting with the relationship among government, enterprises and non-governmental organizations. Peters (2001) put forward the marketization of community governance and held that market governance could break the government monopoly of public service and public management, thus citizens' option rights could be expanded. Some scholars put forward public participation in "smart growth" in order to promote the development of cities and reduce contradiction in community (Laura Pavlot \& Hugh S. Gorman, 2013). These studies reflected the importance of dealing with the relationship among the multiple subjects in community governance, so it was needed to put forward appropriate community governance proposals which adapted to the country according to the country's own national conditions.

Through literature review, we can know that the subject, such as NIMBY, social capital, and community governance has achieved some research results. However, there are few cross research among them and no research putting the three subjects together. Meanwhile, there are a lot of things behind NIMBY that can be used for reference to community participatory governance. Therefore, it is hoped that through the perspective of NIMBY and referring some experience, social capital based on trust will be used in community governance, so as to take advantage of participatory governance and put multi-subject social governance into practice.

\section{Social Capital Issues behind NIMBY}

\subsection{NIMBY Conflict Case Studies}

\subsubsection{America}

There were few conflicts when NIMBY facilities, such as landfills, chemical plants or even nuclear power plants were set in America at early times. But after the 1980s, the situation changed dramatically. In 1984, a private landfill located in Maricopa in Arizona was facing closure. Local residents agreed to build a landfill, but the divergence is the site of the 
building. At first, each location selected by the Maricopa authority was opposed by the public. Later, the authority put public participation in the process of decision-making and established Citizens Advisory Committee made of the municipal authority, local residents, farmers groups and other interest group. After two years of efforts, the government and the public gradually reached a consensus on many controversial issues, and finally decided the location in December, 1988. Thus, the northwest regional landfill was built up.

During 1980 to 1987, 81 facilities have applied for building plants in the United State, but the result was only 6 successfully constructed and operated. The main reason for this situation is that in the past few decades, there has been a great increase in people's public awareness and environmental protection consciousness, but there has been no public participation in the program. Hence, builders of facilities have paid attention to public participation. In 1990, the United States held a national seminar on the establishment of facilities, summarized the reason for high failure rate in the past ten years, and developed a set of facilities setting credo. The first rule was to "design a broad-based participatory process". At the same time, they did not promote the form of administrative orders, but encouraged the use of the voluntary program to get the acceptable site and considered the competitive site selection process. Such a voluntary and competitive process, to some extent, returned the power of site selection to the community. They measured the pros and cons of the facilities setting by themselves, so as to decide whether to accept the construction of facilities. Because it was more democratic and equal, it was welcomed by general public.

Kunreuther by analyzing successful cases occurred in the United States discovered the important factors that prompted community to accept the facility setting were extensive public participation and community residents' full trust to facilities. To begin with, America merely used economic compensation to solve problems but it couldn't solve them fundamentally, leading to the phenomena of NIMBY still. On the one hand, because this way would bring economic pressure to the government and related enterprises, finally the project was still likely to face failure due to shortage of funds and poor management. On the other hand, it was difficult to meet people's need only by compensation. Depressed mood would intensify contradictions, lead to conflict and influence social order. Therefore, the old method was gradually replaced by public participation and competitive site selection. They made information transparent, constructed trust among the public, the government and enterprises, used social capital to build an effective platform for communication, solved conflicts by multi-subject participation, and fully demonstrated the people's subject status. At the same time, the government coordinated and guided every subject, and then fundamentally solved the NIMBY conflicts.

\subsubsection{Japan}

Japan as the world's first country to apply waste incineration technology, has more than 1400 waste incineration furnace. More than $70 \%$ of garbage is going to be put in the incinerator. The data is ahead in the world. The way that they avoid people's opposition is transparent information and public participation.

Musashino municipal incineration plant in Tokyo was built in densely populated urban areas. One of the most important experiences to avoid the NIMBY conflict was public participation in site selection. Details were as follows: environment committee made of the experts and representatives of citizen recommended some persons to participate in the site selection preparation meeting. Each district had its own representatives to participate in the meeting. If they could not select an address within a year, it meant that citizen did not have the ability to make a choice. Then they had to accept the location selected by mayor. Citizen agreed with this plan. After the vote in site selection preparation meeting, four places were included in candidates, including the mayor's initial site selection program. Subsequently, the location selection got into the second stage. Experts, general public representatives and resident representatives of the four candidate sites, in total 35 people, formed another committee and then made a decision.

Daniel through the empirical study and comparative study of nearly 500 cases in Japan, found community with more social capital and closer connection was easier to mobilize and organize NIMBY protest movement. The social development level of local civil society influenced the function of the facility which had been set successfully. The stronger the cohesion of local civil society was, the smaller the possibility of success of the project was. However, if we made the information transparent and encouraged the public to participate in decision-making, social capital could be used well. Through the game among the masses, we could reduce the direct conflict between the masses and the government, implement the facilities project and improve the relationship between the public and the government. Japan's experience showed that they fully used public participation, so that people had more right to talk. Reducing the government's direct leadership could effectively achieve the project. But due to people's large autonomy in the practice of Japan, it might cause the decision was not perfect and scientific. At the same time, in the process of the game between the public and the government or among the public, there might be conflicts. Therefore, social capital based on trust was needed to coordinate and support the game, so as to reduce unnecessary suspicion, misunderstanding and conflict. 


\subsubsection{China}

On May 10, 2014, near Zhongtai village in Yuhang district, Hangzhou, a small part of persons encouraged by criminals closed the provincial highway and expressway, which once caused traffic disruption. Some people even took the opportunity to damage vehicles and hit police and innocent persons. The reason for this incident could be traced back to April 2014. The government in Hangzhou expressed a garbage incineration power plant would be built at Zhongtai village in Yuhang district, which brought worry and resentment to the masses.

Later, Yuhang authority announced, in the absence of the performance of the statutory procedures and people's support, they wouldn't start. In the early period of implementation process, they would invite local people to participate in the whole process and fully listen to the views of the masses to ensure people's information and participation right. Meanwhile, in order to gain public understanding and support, the local government organized experts and representatives in fields of urban construction, planning and environmental protection to have a dialogue, and invited the national waste incineration and treatment experts to answer questions the public concerned. Vice Mayor of Hangzhou Xu Liyi also said that they would ensure people's right to know during the entire process and must meet most people's interests.

The emergence of NIMBY phenomenon in China was relatively late and the related processing mechanism was not perfect. Although the public participation was carried out in many times nominally, it was still dominated by the government. The extent of public participation was largely determined by the attitude of administrative department. The process of the public participation was short, the scope was small and the degree is shallow, which caused that the participation effect was not ideal. Subsequently, a series of documents in the promotion of public participation was published, such as "the Interim Procedures of Environmental Impact Assessment of Public Participation" (2006), "Environmental Information Disclosure Procedure (Trial)" (2008), etc.. But how to achieve more effective public participation is the direction of effort of the government in the future.

The rumor stops in good faith, instead of in one-way information transmission. Social capital based on trust is a good way to deal with social contradictions. Equal multi-subject participation can truly reflect the justice of decision-making and effectively avoid conflicts.

\subsection{Inspiration for Community Participatory Governance from NIMBY}

\subsubsection{Have Public and Transparent Information to Ensure Civil Rights}

The modern social conflict is a kind of confrontation between rights and actual supply, politics and economy, civil rights and economic growth. The problem of NIMBY is actually a game between power and right. Modern social information is huge, but the conflict caused by information asymmetry has brought society an unstable situation. Therefore, information disclosure and transparency is the first step to solve the problem of NIMBY. Information transparency guarantees the public's right to know. Through the implementation of further specific programs, people's rights and interests have been protected. During this period, government's credibility has been enhanced, so as to promote cooperation between the government and the public, reduce administrative costs, improve administrative efficiency, avoid the occurrence of NIMBY conflict, and maintain social stability. It also can establish reciprocal norms for the problem, so that people and the government are able to know the interests of their own, but at the same time, each subject communicate with others within a reasonable standard range, which is one of the aspects of social capital practical application.

\subsubsection{Bring Public Participation in Decision-making to Exert Citizens' Initiative}

Public participation is an important part to avoid NIMBY. Unclear decision-making process leads to the passivity of the public and the surface of democracy. Substantially, citizens are unable to participate in specific practical activities and express their views and aspirations. Relevant rights and interests also have been violated, so as to sow the seeds for NIMBY conflict. Therefore, let the public take part in the discussion, decision-making, and even the follow-up supervision, so that the public can feel their own subjective status and play their subjective initiative, which is beneficial to the construction of civil society.

Public participation makes the public, government, enterprises and other subjects form a participatory network, which contributes to the formation of social networks and is beneficial to play their respective roles for each subject. Meanwhile, it is helpful for equal communication and consultation. It also can strengthen contact among them, enhance acquaintance and understanding, develop the superiority of social network resources of social capital and promote effective solution of the problem.

\subsubsection{Pay Attention to the Positive Interaction to Build Social Trust}

One of the vital reasons is the mutual distrust between citizens and the government. Citizens do not trust the guarantee 
and commitment that the government made while the government also does not believe that citizens can make scientific decision-making and planning. As a result, it leads to formal "democracy" decision, external information block, and public discontent and protest.

Social capital is an effective way to alleviate the contradiction between government and citizens. Using social capital rebuilds social trust, so that people are no longer "atomic" but in the state of mutual contact and mutual trust. Social capital promotes the change of society from "stranger society" to "acquaintance society" and makes society operate benignly and develop well.

\section{Measure Suggestions}

\subsection{Build a Platform of Information Disclosure and Ensure Community Information Flow}

Rebuilding trust capital is the premise of improving community governance. With the development of digital information technology, the new media technology, which is represented by mobile phone and Internet, has broken the traditional information exchange and communication mode. New media's interactivity and immediacy, mass and sharing, multimedia and hypertext, personality and socialization, and other characteristics, have attracted the public's attention, so that the use of new media continues to rise. The public contact with other people through the media, so new media become a new tool and new platform for people to communicate with others. Therefore, community can use micro-blog, We Chat, blog, podcast and forums or establish their own website to publish community construction information, community activities, notification, etc., set up a comprehensive set of community public information platform, and timely announce community governance information for community members, so that the community information is public, transparent and unobstructed. Because of strong interaction and wide use of new media, community members can break through time and space constraints, express their ideas, opinions, suggestions, etc., and actively participate in community governance, so as to mobilize community members' initiative, which reflects community members' subject status in community participatory governance.

\subsection{Establish the Standard of Community System and Improve Members' Identification Degree}

The reconstruction of the reciprocal norm is the guarantee of improving the way of community governance. Because of the complexity of community members, the cognition and value judgment also vary from person to person. Therefore, the premise of community participatory governance is forming a common value standard system, so as to provide insurance for the healthy operation of social capital. Community system and norms provide common value standard for community members. It is beneficial to improve the members' identification to community, which is conducive to the orderly development of community governance.

Community system standard should be systematic. Problems that exist or will show up in community governance should be illustrated carefully. Construct comprehensive and systematic community institution, and make community work rule-based in every aspect, so as to ensure the efficiency and the order of follow-up community governance work. However, mechanical or rigid treatment ways should also be avoided.

Community system standard should be dynamic. Combined with the community present situation and the trend of social development, we should constantly improve the community governance systems and norms, keep pace with the times, update minds timely and have the spirit of innovation, instead of being fixed and conservative.

Community system standard should be normalized. We should put community governance systems or norms into practice actively after publishing them, rather than keep them a mere scrap of paper and be an armchair strategist. To avoid staying superficial or formal, normalization is a good way to integrate members' thought, cognition, behavior and reflects the spirit of the community.

\subsection{Build Community Participation Network and Strengthen Mutual Connection between Residents}

With the continuous development of modern society, people's life tempos become more and more quick. Individuals gradually become atomic individuals, and the whole society changes from "acquaintance society" into "stranger society". People only care about their own things and have these kinds of attitudes, such as "let things drift if they do not affect me personally" or "the less trouble the better". As a consequence, the situation that no one helps an old man who falls down may occur. Neighbors don't know each other and are completely isolated from each other all their lives. There is no contact with each other, let alone warmth between them. An old saying goes, "a near neighbor is better than a distant relative." But things have changed now.

Community is the place where residents live. It is one of the most basic social components, and it is closely related to the residents. Community governance should focus on the cultivation of community members' sense of belonging and identification, promote the participation and communication among residents by developing a variety of community theme activities, cultivate residents' good impression on the community, enhance residents' awareness and concept of 
citizens, and increase the contact and affection among residents, thus we can form a network that community members are able to communicate with each other, so as to form the whole community participation network.

At the same time, we can also make good use of the new media to promote mutual connection among community members and construct community participation network. The information sharing and convenient information exchange are helpful to the consensus of the group, the establishment of the relationship circle among members of the society, and the elimination of group polarization phenomena. So taking advantage of the new media can promote the interaction among community members, enhance the sense of belonging and cohesion of community members, and promote the construction of community.

\subsection{Perfect Community Autonomy Organization, and Improve the Efficiency of Community Governance}

As one of the subjects of community governance, community autonomy organizations play an important role in the process of community residents' participation in community governance. It is beneficial to improve community residents' citizen consciousness and the quality and efficiency of community governance.

Community autonomy organizations can perfect their organization development through regular meetings, opinion gathering, risk assessment, full supervision, and performance inspection. Meanwhile, through a series of operational mechanisms, multivariate community governance subjects can integrate a common force to complete the task of community governance. Residents committee, the proprietors committee, property management committee, mutual aid organizations which are established through residents' spontaneity and other community autonomy organizations, under the perfect rules, ought to coordinate with each other, trust each other, and maintain information flow to have more communication, more exchanges, mutual dependence and resource sharing. Via dialogue and consultation, integrate the value cognition, establish the basis of mutual understanding, and enhance the complementation among resources, information and advantages, in order to achieve the resolution of contradictions and conflicts, improve the efficiency of overall community governance, and form a win-win situation. In the process of devolution, we are supposed to take full account of various factors to avoid that power and resources are too concentrated on one subject. The power and resources are distributed to different governance subjects in community, and then form a relationship structure of mutual dependence, mutual sharing, mutual interaction, sharing responsibility and reciprocity, so as to ensure the equality of status of each subject in community governance network, the horizontality of coordination relationship, the communality of decision-making and the cooperation of action.

\subsection{Establish Civil Value Culture and Promote the Construction of Harmonious Community}

Any kind of social construction process or social governance system needs a set of value culture system which adapts to it. Value culture, as a kind of social capital, is showed as value identification and attribution, which has the dual function of determining direction and providing power in social construction and social management system. It is not only the principle of social relations adjustment, but also the norm of system construction and standard action. Forming systemic and integral community members' value culture in community is beneficial to the establishment of community trust and cohesion among community members, so that social capital can effectively be applied to community governance.

One of the most important steps is citizen's cognitive identification and internalization. Community members need to absorb and internalize the value culture which is advocated by the community, then apply it to their own practice. This reflects civic endogenous culture value appeals, which are linked with the essence of cultivating modern civil society. They both aim at reconstruction of social internal capital, construction of endogenous social order, and exploration of endogenous motivation of social development in China. Therefore, start from every community citizen, establish their own value culture, thus form huge force of social capital and promote the construction of harmonious community.

\section{Conclusion}

We have seen the importance of social capital and participatory governance to social governance from NIMBY, so we need to pay attention to them and make good use of them. Social capital is the glue of community governance and the basis of cooperation among community governance subjects. High social capital stock represents that the level of trust among community governance subjects is high and there are more opportunities for cooperation and consultation, which directly affect operating conditions of community governance main network. To increase the stock of social capital needs to expand the channels of community participatory governance for community residents and community organizations and improve the level of participation. All in all, the more opportunities community residents and community organizations have in community participatory governance, the better it is to increase the stock of social capital. At the same time, social capital promotes community governance in turn. Now, the development of community governance in China is still not mature, so we can refer to experience of other countries or other social governance issues in China, foster strengths and circumvent weaknesses, promote the use of social capital in community participatory governance, and accelerate the construction of harmonious community and harmonious society in China. 
In this research, a Chinese model of social capital application in community participatory governance has been constructed according to Chinese national condition. Hope it will be beneficial to China in transition and give a reference for other countries which need to develop community governance. Because of the differences in political, cultural and other factors, the specific measures of community participatory governance may vary from country to country. Therefore, the way of implement should be changed according to the national conditions, so as to adjust measures to local conditions. Moreover, it needs to be further studied in the future whether the model is suitable for other countries.

\section{References}

Bourdieu, P. (1980). Le capital social:notes provisoires. Actes De La Recherché En Sciences Socials, 1(31), 2-3.

Chen, H., \& Ding, H. (2014). Social governance path innovation: the perspective of trust and cooperation order. Jianghai Academic Journal, (4), 228-233.

Chen, S. Y., \& Zhao, G. Y. (2009). A review of literature on "participatory governance". Teaching and Research, (8), 75-82.

Chen, W. T. (2014). Improving social governance mode: exert the basic function of public participation. Journal of the Party School of CPC Jinan Municipal Committee, 1, 75-78.

Daniel, A. (2008). Location, location, location: selecting sites for controversial facilities. The Singapore Economic Review, (1). http://dx.doi.org/10.1142/S0217590808002884

Emilie Travel Livezey. (1980). Hazardous waste. Christian Science Monitor.

Findlay, T. (2014). Social capital and local governance regimes: early childhood development roundtables in British Columbia. Innovation Journal, 19, 1-20.

Guo, F. (2015). How does America deal with NIMBY. Decision-Making, (5), 74-75.

Guo, X. C., \& Dai, K. (2013). A review of the research on domestic citizen participation in the last ten years. Academic Research, (6), 29-35.

Hartmut Schneider. (1999). Participatory Governance for Poverty Reduction. Journal of International Development, 11(4), 521-534. http://dx.doi.org/10.1002/(SICI)1099-1328(199906)11:4\%3C521:AID-JID599\%3E3.0.CO;2-J

James, S. C. (2008). Foundations of social theory. Beijing: Social Sciences Academic Press.

Kunreuther, H., Fitzgerald, K., \& Aarts, T. (1993). Siting Noxious Facilities: A Test of the Facility Sitting Credo. Risk Analysis, 13(3), 301-318. http://dx.doi.org/10.1111/j.1539-6924.1993.tb01082.x

Laura, P., \& Hugh, S. G. (2013). Environmental reviews and case studies: public participation and smart growth in Silver Spring, Maryland. Environmental Practice, 15(2), 156-168. http://dx.doi.org/10.1017/S1466046613000069

Li, Z. Z. (2013). NIMBY and the implications to social management. Journal of Zhengzhou University (Philosophy and Social Sciences), 46(6), 23-27.

Lindén, A., Rapeli, L., \& Brutemark, A. (2015). Community attachment and municipal economy: public attitudes towards wind power in a local context. Environmental Science \& Policy, 54, 10-14. http://dx.doi.org/10.1016/j.envsci.2015.06.005

Meng, D. K., \& Zhu, J. Y. (2010). Exceed Japan (vol.2)—waste incineration: learn from Japan, but not learn all things. http://www.infzm.com/content/52134

Michael, O'H. (1977). Not on my block you don't: facility siting and the strategic importance of compensation. Public Policy, (4).

Qian, H. M. (2011). Social capital: A study on the resource allocation method based on trust. Modern Management Science, (2), 88-90.

Robert, D. P. (2015). Making Democracy Work: Civic Traditions in Modern Italy. Beijing: China Renmin University Press.

Robin, G., \& Howard, K. (1990). Successful Siting Incentives. Civil Engineering, 60(4), 73-75.

Ruth, B. M. (2000). Consequential Utilitarianism: Addressing Ethical Deficiencies in the Municipal Landfill Siting Process. Journal of Business Ethice, 26(4), 289-306. http://dx.doi.org/10.1023/A:1006345600415

Shi, B., \& Wu, X. X. (2009). Analysis of social capital's function in community governance-from the angle of malfunction dilemma. Scientific Decision Making, (7), 83-89. 
Shi, Y., \& Tu, H. M. (2010). Basic social cooperation governance: exploration of a feasible path to improve the Chinese characteristic citizen governance. Social Science Research, (3), 48-54.

Vittes, M. E., Pollock, P. H., \& Lilie, S. A. (1993). Factors Contributing to NIMBY Attitudes. Waste Management, 13(2), 125-129. http://dx.doi.org/10.1016/0956-053X(93)90004-G

Wang, H. (2014). Study on urban community governance from the perspective of NIMBY .Management Observer, (31), 185-188.

Yan, H., \& Su, R. N. (2007). Analysis on the transformation of urban community governance structure in China. Journal of the Party School of Fuzhou, (5), 35-38.

Yan, J. R. (2010). Community governance and social capital investment - the theoretical explanation of community governance innovation in China. Tianjin Social Sciences, (3), 59-64.

Yuan, Z. L. (2011). Participatory democracy theory research. Beijing: China Social Science Press.

Zhang, H. X. (2014). Research on the innovation path of community participation mode in new media context. New West, (17), 12-15.

Zheng, H. S., \& Huang, J. L. (2012). New trends of social management and community governance in China. Gansu Social Sciences, (6), 1-8.

Zhu, T. (2014). "NIMBY"conflict and public participation in the era of "social governance". Urban and rural governance and planning reform-symposium of Chinese urban planning annual meeting (11-Planning implementation and management).Hainan, China: China Urban Planning Society.

\section{$(\mathrm{cc}) \mathbf{E Y}$}

This work is licensed under a Creative Commons Attribution 3.0 License. 